courses. We assessed changes in mental health (GAD-7) and disease control (IBD-Control) using patient-reported outcome measures delivered through the app.

Results Patients scored significantly higher on the IBD-control questionnaire following completion of either the 4 or 5-day courses, indicating that they feel more control over their disease following the intervention (median score 5 vs 8.5, $\mathrm{p}<0.002$ ). We observed non-significant improvement in selfreported anxiety and depression levels, a 17\% improvement in self-reported wellbeing, along with an increased level of physical activity, and feelings of social connection.

Conclusions These measurable improvements following a short course highlight the need for supported self-care for people with IBD during uncertain times. Future work will investigate the effectiveness of non-lockdown-related courses on self-management of IBD in app users.

\section{PTH-17 THE VIRTUAL REFERRAL TRIAGE CLINIC - A BENEFICIAL CHANGE IN PRACTICE?}

Sue Priestley, A Liaros*. St Helens and Knowsley Teaching Hospitals NHS Trust, Prescot, UK

\subsection{6/gutjnl-2021-BSG.332}

Introduction Due to the COVID 19 pandemic gastroenterology elective activities were suspended in March 2020. When elective services were reinstated, social distancing imposed within the Trust resulted in a significant loss in traditional F2F (face to face) clinic capacity. A Consultant led Virtual Referral Triage Clinic (VRTC) was introduced with the aim to ensure ongoing patient care within the confines of the pandemic. Our objectives were to review of all non-2WW clinic referrals to determine and maintain appropriate, safe patient management whilst reducing hospital attendances.

Method A Gastroenterologist reviewed all non-2WW referrals within 24 hours of receipt of referral. VRTC sessions (4 hours) were provided 5 days/week. The potential outcomes of triage were: redirect to a more appropriate speciality, direct to straight to test (STT), discharge with 'advice and guidance' to the patient and/or GP and arrange a F2F appointment or telephone appointment (prioritised as urgent or routine). Data was collected in Excel and analysed.

Results Above 1900 non-2WW referrals between 01/04/2020 $31 / 10 / 2020$ were received. Complete data was available in 1448 cases allowing detailed analysis. $88.9 \%$ of referrals were from primary care, the remainder from secondary care. Below are the VRTC outcomes.

$9 \%$ were redirected to another speciality; $68 \%$ of those to colorectal or upper GI surgery. 38\% were directed to a telephone clinic. $25.3 \%$ went STT, the majority for endoscopy, radiology and/or bloods tests e.g. parenchymal liver screen. 100 of the STT cases were reviewed; 79\% were discharged or discharged as failed to attend their STT investigation. A total of $42.4 \%$ were redirected or discharged at triage or following STT investigations. Overall $86.3 \%$ of patients were managed without a 'new' patient F2F clinic appointment. This equates to approximately $155 \mathrm{~F} 2 \mathrm{~F}$ clinic sessions (8 patients/session). It required 105 VRTC sessions to complete work, therefore saved 50 clinic sessions.

Conclusions The challenges include the ability to access enough information to enhance decision making at triage and the need to formalise in consultant job plans so that the process can continue. There is little evidence that neither patients

\begin{tabular}{lll} 
Abstract PTH-17 Table 1 & & \\
\hline Triage Outcome & Number & $\%$ \\
\hline STT & 368 & 25.3 \\
Discharged with & 196 & 14.0 \\
A\&G & & \\
F2F Routine & 469 & 32.0 \\
F2F Urgent & 84 & 6.0 \\
Telephone & 168 & 12.0 \\
Routine & & \\
Telephone Urgent & 34 & 2.0 \\
Redirected & 129 & 9.0 \\
\hline
\end{tabular}

nor GPs are significantly dissatisfied with this change in practice, with low rates of re-referral or complaint. It also does not appear patients come to harm or are mismanaged by this approach but may need longer term follow-up. There are obvious benefits: redirection of patients to more appropriate care, earlier diagnosis in those directed STT and a large reduction in unnecessary patient attendances with a subsequent significant reduction in the need for F2F clinic capacity.

\section{PTH-18 ELIGIBILITY FOR A STRAIGHT TO TESTCOLONOSCOPY EXAMINATION IS BEST JUDGED BY SECONDARY CARE}

Yen Yeo, Touqeer Raja, Alex Ball. Sheffield Teaching Hospitals NHS FT, Sheffield, UK

\subsection{6/gutjnl-2021-BSG.333}

Background Straight to Test (STT) is the delivery of an appropriate diagnostic service without the requirement for the patient to first attend an out-patient clinic hospital appointment. A STT colonoscopy pathway was initiated for 2 weekwait (2ww) colonoscopy examinations at Sheffield Teaching Hospitals in March 2019. This pathway is associated with a reduced time to colonoscopy and therefore cancer diagnosis or exclusion. To select appropriate patients, General Practitioners were asked whether patients were suitable for a STT examination on the referral form. Patients judged inappropriate for a STT examination were seen for a face to face consultation prior to colonoscopy. This study assesses whether there was justification for excluding these patients from a STT examination.

Methods The clinical notes and investigations of patients that were referred to the Lower GI pathway between May and July 2019 and judged inappropriate for a STT examination were reviewed by two clinicians. Demographic information, clinical symptoms, FIT results and investigations were performed. Potential reasons for exclusion form a STT examination were assessed and categorised. The incidence of colorectal cancer was also assessed.

Results During the study period 104/985 (11\%) patients were judged to be inappropriate for a STT examination of which $42 / 104(40 \%)$ were male with a mean age of 69 (range 42 $89)$ years. Reasons for referral included assessment of bleeding (23), change in bowel habit (61), anaemia (46), positive FIT test (10) and abdominal pain (23) with many having overlapping symptoms. There was justification for exclusion from a STT examination in 70/104 (67\%) patients: 37 had significant comorbidities of whom 3 had dementia, 13 due to patient choice, 8 were frail, 5 did not speak English, 3 were for 
assessment of incontinence although should probably not have been on a cancer pathway, 2 had a previous failed colonoscopy and 1 had learning difficulties. There was a single cancer in this patient group. Despite having a reasonable justification for exclusion from a STT examination 27/70(39\%) patients still went on to have an endoscopic examination. We found no justification for exclusion from a STT examination in $34 / 104(33 \%)$ patients of whom $31(91 \%)$ went on to have a colonoscopy following a clinic review and two of these patients had a colorectal cancer.

Conclusion Two-thirds of patients judged inappropriate for a STT examination were appropriately identified by primary care referrers. However, there was no justification for why a third of patients were excluded and the large majority of these proceeded to a colonoscopy. This data supports secondary care providers being the key decision makers in allocation of patients for $2 \mathrm{ww}$ STT colonoscopy examinations.

\section{PTH-19 ASSESSING THE IMPACT OF SBAR PROFORMA AND GI BLEED ROTA IN MANAGING VARICEAL BLEED PATIENTS}

${ }^{1}$ Irfan Ullah* ${ }^{2}$ Amir Hazizi Abdul Razak, ${ }^{3}$ Gireesh Kothegal Marimahadevappa, ${ }^{4}$ Peter Neville. ${ }^{1}$ Nevill Hall Hospital, Abergavenny, UK; ${ }^{2}$ Prince Charles Hospital, Merthyr Tydfil, UK; ${ }^{3}$ Prince Charles Hospital, Merthyr Tydfil, UK; ${ }^{4}$ Prince Charles Hospital, Merthyr Tydfil, UK

\subsection{6/gutjnl-2021-BSG.334}

Introduction Variceal bleeding in liver disease is associated with significant mortality and morbidity. Early recognition and appropriate interventions are crucial in managing patients with acute variceal bleeding.

Methods Patients presented with acute variceal haemorrhage to Prince Charles Hospital and Royal Glamorgan Hospital were retrospectively identified using our endoscopy database. Four locally agreed key performance factors including early endoscopy within 24 hours, use of prophylactic antibiotics, documentation of Glasgow Blatchford Score (GBS) and correct identification of upper gastrointestinal bleed (UGIB) on initial presentation were assessed in patients presented between February 2018 to February 2019 in the initial audit cycle.These factors were audited again for patients from March 2019 to December 2019, after the introduction of SBAR proforma and out-of-hours (OOH) GI bleed on call rota.

Results A total of 31 patients were included in initial audit and 13 patients in the reaudit.In the first audit it showed that compliance with endoscopy within 24 hours was 96\%, prophylactic antibiotics was 74\%, GBS documentation was 55\% and identification of upper GI Bleed on admission was $100 \%$. After the introduction of UGI bleed SBAR proforma and $\mathrm{OOH}$ GI bleed on call rota, the repeat audit of the same factors showed improvement in GBS documentation to $92 \%$ and prophylactic antibiotics use on admission to $85 \%$ while UGIB identification on admission remained 100\%. Endoscopy within 24 hrs of admission was $85 \%$, but this was due to the risk benefit review of a case and the need for urgent endoscopy by the team.Endoscopic procedures done $\mathrm{OOH}$ initially was $22 \%$ which rose to $31 \%$ after introduction of UGI bleed on call service. Additional information also showed that SBAR proforma was used in $62 \%$ of cases. It also showed better use of the in hours endoscopy services, as more cases were discussed appropriately with the on call Gastroenterologists.

Conclusion This audit concludes that there was better documentation of GBS, use of prophylactic antibiotics, appropriate early recognition and management of variceal bleeding by using available endoscopy service and the appropriate use of on call service for these patients, who generally have significant morbidity due to their underlying liver disease. We recommended that repeating the audit cycle in 12-24 months would be valuable to see whether these improvements are sustained and to reassess the long term benefit of GI bleed on call service in effectively managing these patients. We also suggest that we should aim to achieve 100\% compliance in these performance factors along with appropriate management of associated liver disease.

\section{PTH-20 THE IMPACT OF COVID-19 PANDEMIC ON UPPER GI AND HEPATO-PANCREATO-BILIARY CANCERS - DIAGNOSIS AND STAGING}

${ }^{1,2}$ Ee Xuan Ngeyu* ${ }^{*}$ Palaniappan Jeyam-Suresh, ${ }^{1,3}$ Anjan Dhar. ${ }^{1}$ County Durham and Darlington Nhs Foundation Trust, Darlington, UK; ${ }^{2}$ Newcastle university Medical School, Newcastle, UK; ${ }^{3}$ School of Health and Life Sciences, Teesside University, Middlesbrough, UK

\subsection{6/gutjnl-2021-BSG.335}

Introduction Cancer Charities and the BSG Endoscopic Section predict an increased GI cancer related mortality arising from reduced endoscopic activity and delays to cancer diagnosis during the COVID-19 pandemic. Initial modelling reports suggest up to a $58 \%$ reduction in weekly GI cancer detection. A real world analysis of the adverse impact of COVID-19 on Upper GI and hepato-pancreato-biliary (HPB) cancers has not been reported so far.

Aims and Methods Patients with a new diagnosis of upper GI (oesophageal and gastric) or HPB cancer at County Durham over two 4-month periods of June-September 2019 (preCOVID) and 2020 (peri-COVID) were selected for this analysis. Data collected from electronic patient notes and Somerset Cancer MDT electronic database included: 1) patient demographics and co-morbidities; 2) Pathway parameters - duration of symptoms prior to presentation, 2WW timeframes, interval between referral and endoscopy, performance status, elective vs emergency presentation, and 3) Tumour parameters - cancer site, TNM staging. 90-day mortality, where available was also recorded.

Results 163 patients were included - 82 patients in preCOVID (2019) and 81 in peri-COVID (2020) cohorts. In the pre-COVID group, 25 were oesophageal cancers, 8 gastric, 21 pancreatic, and 26 liver cancers. In the peri-COVID group, 31 were oesophageal cancers, 13 gastric, 17 pancreatic, and 18 liver cancers. An absolute increase of $17.5 \%$ was seen in emergency presentation of UGI cancers to A\&E in 2020 $(35.8 \% \mathrm{v} 18.3 \%)$. In the peri-COVID group, $87.1 \%$ of patients had advanced (TNM staging of T3/4, N2 or M1) oesophageal and gastric cancer, $7.1 \%$ more than in preCOVID group. A similar increase of $6.4 \%$ was identified in metastatic pancreatic cancers $(58.8 \%$ in $2020 \mathrm{v} 52.4 \%$ in 2019). There was a significant reduction in the number of endoscopies performed within 3 weeks of referral - 56.8\% pre-COVID, compared with only $20 \%$ peri-COVID. There was no significant difference between 60-day mortality (preCOVID 25.61\% and peri-COVID group 25.91\%) and 90-day mortality $(34.15 \%$ and $32.10 \%$ respectively), between the two groups.

Conclusion Although the impact of COVID-19 on endoscopy activity resulted in a $17.5 \%$ increase in emergency presentations of UGI and HPB cancers and nearly $10 \%$ increase in 